\title{
CLINICAL AND PATHOLOGICAL DIFFERENCES BETWEEN THE PYOGENIC GRANULOMA AND LOBULAR CAPILLARY HEMANGIOMA IN THE ORAL CAVITY: A SCOPING REVIEW
}

\author{
Jonas Ver Berne', Erich J. Raubenheimer ${ }^{1,2}$, Reinhilde Jacobs ${ }^{1,3}$, Constantinus Politis ${ }^{1,4}$ \\ 1OMFS-IMPATH, Catholic University Leuven, Belgium \\ ${ }^{2}$ Department of Oral Pathology and Oral Biology, School of Dentistry, Faculty of Health Sciences, University of Pretoria, Pretoria, South Africa \\ ${ }^{3}$ Department of Dental Medicine, Karolinska Institute, Stockholm, Sweden \\ ${ }^{4}$ Department of Oral and Maxillofacial Surgery, University Hospital Leuven, Belgium
}

\begin{abstract}
Since initially described, the terms "pyogenic granuloma" (PG) and "lobular capillary hemangioma" (LCH) have undergone a significant change in meaning, which leads to a confusion in the literature. In this study, we attempted to investigate if there are any clinical and pathological differences between the pyogenic granuloma and lobular capillary hemangioma through a scoping review of the available literature. A Medline search has identified 342 articles from 2001 to 2018, from which English papers, case series, retrospective studies, and cross-sectional studies were selected. Data from the articles was charted using appropriate subheadings for the clinical question (e.g., number of patients, clinical features, histology, pathogenesis, differential diagnosis, treatment). Compared to LCH, PG seems to occur more in the middle-aged range, has a clearer predilection for (pregnant) females, and has more frequently a known triggering factor in the history. Histologically, PGs appear as radially organized capillaries (while LCHs have a lobular pattern), have a mixed inflammatory infiltrate (compared to the occasional lymphocytic infiltrate in LCHs), and can undergo fibrosing transformation when chronic, which is not seen in LCHs. Finally, besides basic excision, several other treatment options have been described for both lesions. Since the pyogenic granuloma and lobular capillary hemangioma show clinical and pathological differences, we propose them to be considered as distinct lesions, which makes interpreting the literature much easier.
\end{abstract}

KEY WORDS: pyogenic granuloma, lobular capillary hemangioma, pathology, histology, oral mucosa.

J Stoma 2020; 73, 4: 206-216

DOI: https://doi.org/10.5114/jos.2020.98315

\section{INTRODUCTION}

The pyogenic granuloma (PG) was first described in 1943 [1]. Since then, it is being referred to as a red colored, smooth, or lobulated exophytic lesion on a pedunculated or sometimes sessile base, with a tendency to bleed easily [2]. The term "pyogenic granuloma" is a misnomer, for the lesion is neither "pyogenic" (pus forming) nor is it a "gran- uloma" (an organized collection of inflammatory cells of the monocyte family). Therefore, the term "lobular capillary hemangioma" (LCH) was introduced in 1980 as the underlying histological equivalent [3]. Over the years, the meaning of this term has changed significantly, from a reactive hyperplastic mucosal lesion, granulation tissue, to a benign vascular tumor, which contributes to the confusion in the literature. In 2003, Toida et al. brought

\section{JOURNAL OF} STOMATOLOGY CZASOPISMO STOMATOLOGICZNE

ADDRESS FOR CORRESPONDENCE: Jonas Ver Berne, OMFS-IMPATH, Catholic University Leuven, Kapucijnenvoer 33, 3000 Leuven, Belgium, e-mail: jonas.verberne@student.kuleuven.be

RECEIVED: 17.05.2020 • ACCEPTED: 05.07.2020 • PUBLISHED: 30.08.2020 
some clarity by stating that there are in fact two types of the lesion: the LCH and the non-LCH, each with distinct clinical, histological, and pathophysiological features [4]. However, since the disconnection of these two types, few attentions has been given to it in later research. Discrepancy exists even in the major classifications, where in the WHO 2017 classification of head and neck tumors, the PG and LCH are considered synonyms and are classified as hemangiomas, and in the ISSVA classification (International Society for the Study of Vascular Anomalies, 2018) both lesions are classified as benign tumors. Of course, this adds to the confusion. Despite the limited number of articles on this specific topic, we attempted to offer an overview of any evidence on clinical and pathological differences between the two lesions in the oral cavity, based on a scoping review of the available literature.

\section{MATERIAL AND METHODS}

A literature search was performed in the Medline database using the search terms presented in Table 1, revealing 342 usable articles, excluding non-English articles and articles published before 2001. The articles were screened by title and abstract on relevance. Inclusion criteria were English language and full text availability provided by the KU Leuven. Further exclusion criteria were irrelevant descriptions of the lesion, individual case reports, and reviews. After this selection, 15 relevant studies remained (Figure 1). Areas of interest were formulated (cfr. subheadings) and data was obtained from the articles (Table 2).

\section{RESULTS}

Where possible, the articles were classified according to the type of lesion studied, PG and/or LCH, based on histological descriptions provided. In the following sections, areas of interest are covered to enable distinct description and differential diagnosis of both pathological entities (PG and LCH).

\section{DEFINITION AND SYNONYMS}

A variety of synonyms are used for PG, such as $\mathrm{LCH}$, granuloma pyogenicum, granuloma telangiectaticum, angiogranuloma, epulis gravidarum, and pregnancy tumor

TABLE 1. Medline search term

("granuloma, pyogenic" [Mesh] OR "pyogenic granuloma" [tiab] OR "oral pyogenic granuloma" [tiab] OR "lobular capillary hemangioma" [tiab] OR "granuloma pyogenicum" [tiab] OR "granuloma telangiectaticum" [tiab] OR "angiogranuloma" [tiab] AND ("Mouth" [Mesh])) OR ("Granuloma, Pyogenic/etiology" [Mesh] AND "Mouth" [Mesh]) OR ("Granuloma, Pyogenic/pathology"[Mesh] AND "Mouth" [Mesh]) OR ("granuloma, pyogenic/diagnosis" [Mesh] AND "Mouth" [Mesh]) OR ("granuloma, pyogenic/therapy" [Mesh] AND "Mouth" [Mesh]) OR (("Neoplasms, Vascular Tissue"[Mesh] OR "vascular tumor"[tiab] OR "vascular tumour"[tiab] OR "vascular tumors"[tiab] OR "vascular tumours"[tiab]) AND ("Granuloma, Pyogenic"[Mesh] OR "pyogenic granuloma"[tiab] OR "pyogenic granulomas"[tiab] OR "granuloma pyogenicum"[tiab] OR "granuloma gravidarum"[tiab] OR "pregnancy tumor"[tiab] OR "pregnancy tumour"[tiab] OR "granuloma telangiecticum"[tiab] OR "lobular capillary hemangioma" [tiab] OR “Iobular capillary haemangioma”[tiab] OR "angiogranuloma"[tiab] OR “angiogranulomas"[tiab]))

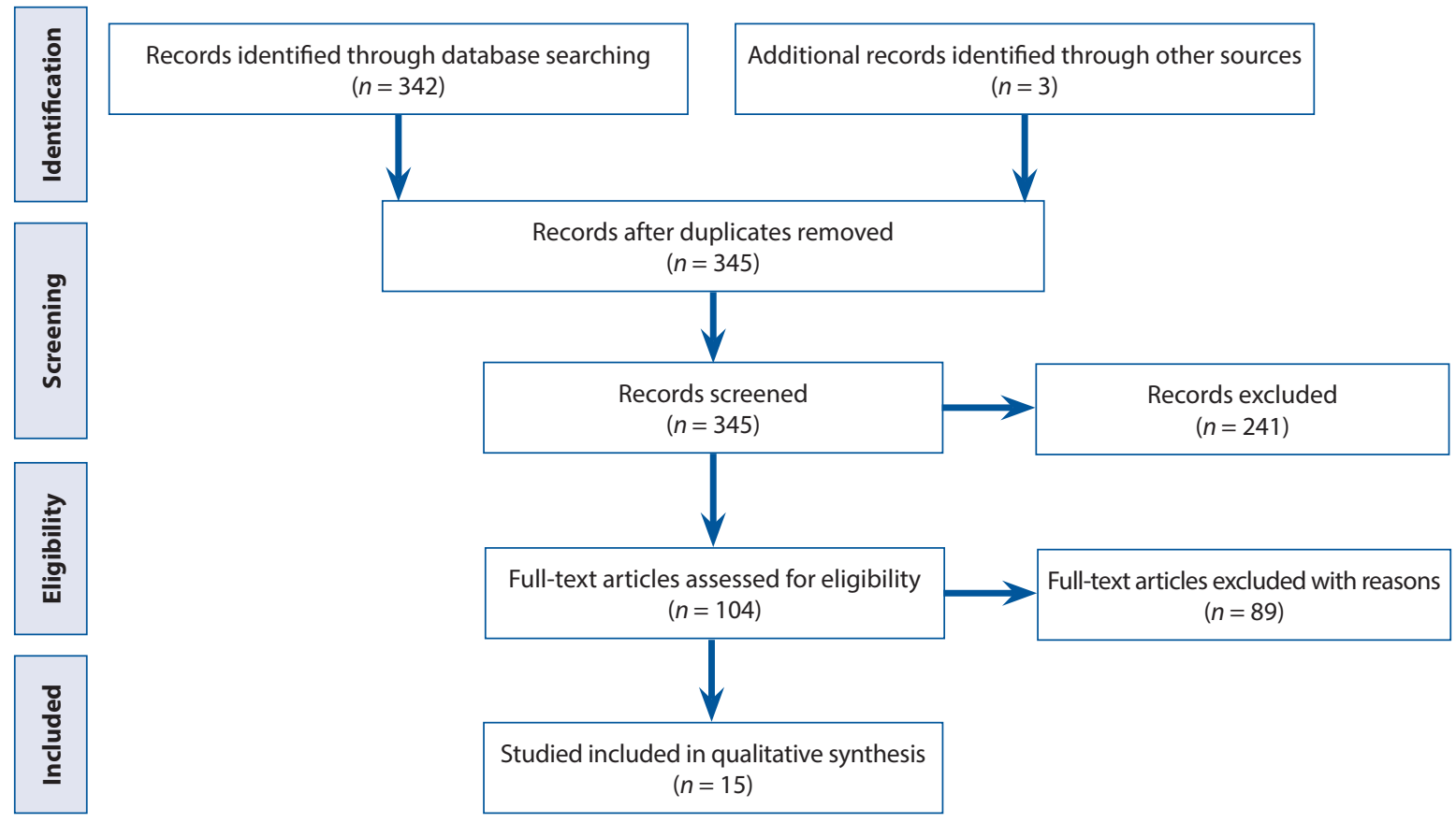

FIGURE 1. PRISMA flowchart on study selection and inclusion 


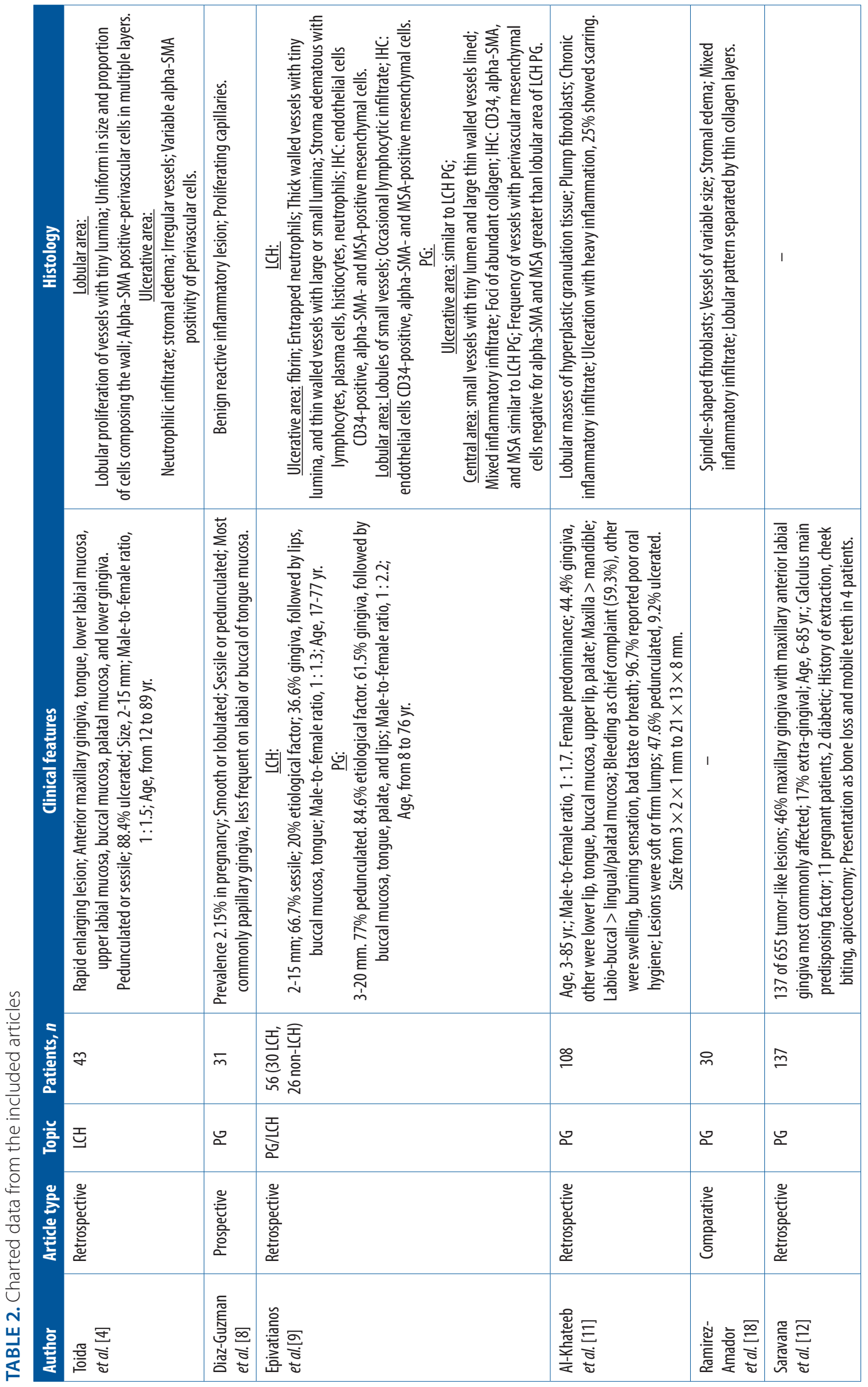




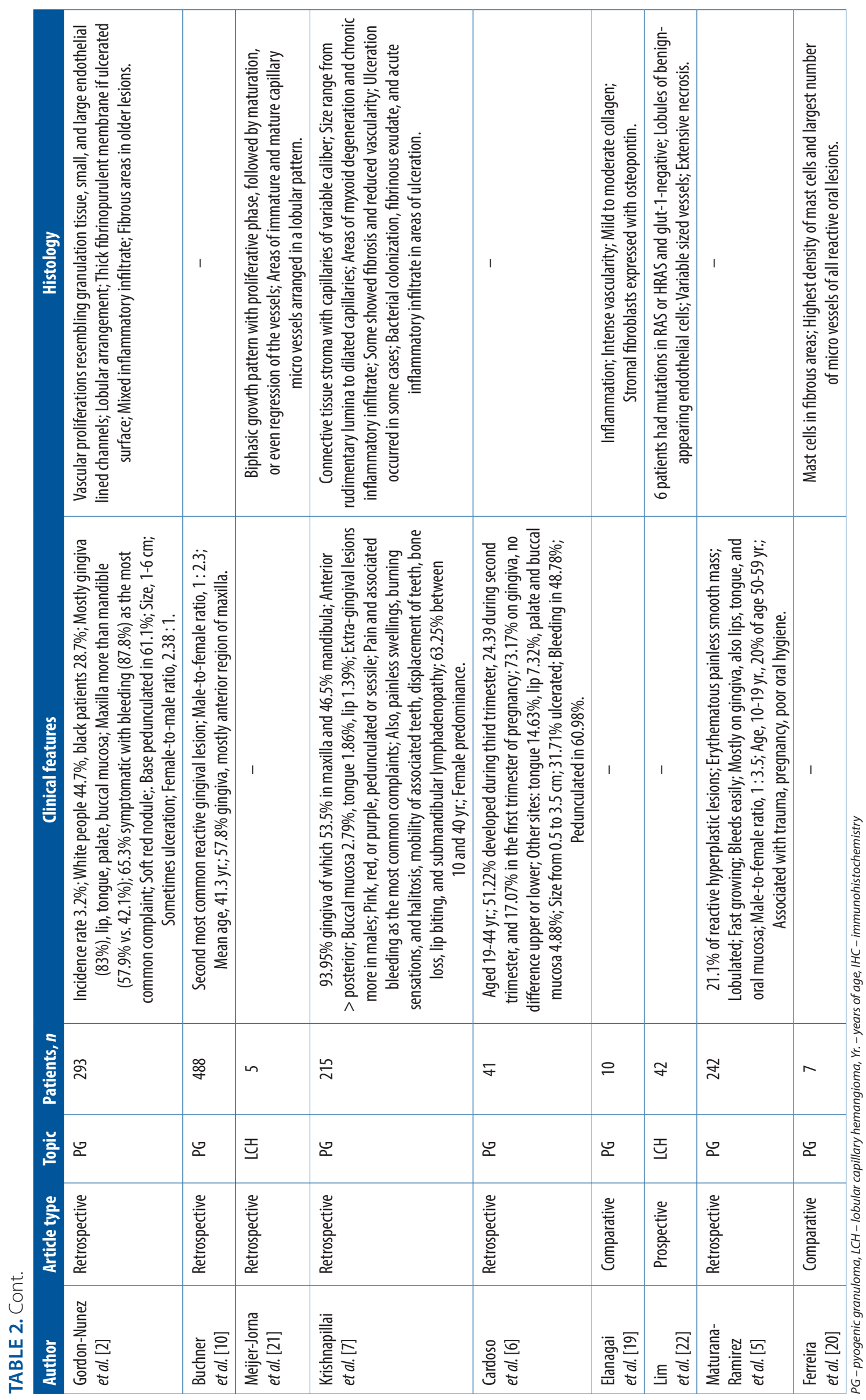



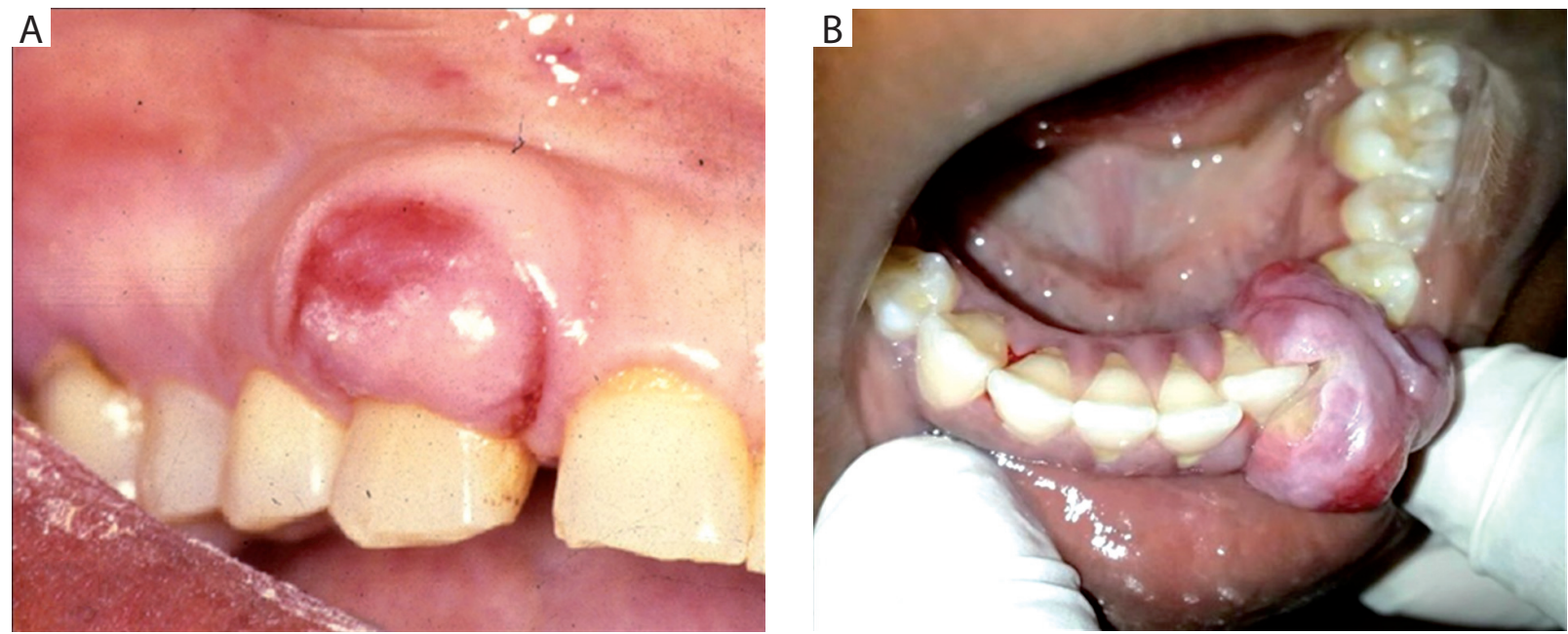

FIGURE 2. Two clinical pictures of pyogenic granulomas presenting as ( $\mathrm{A}$ ) an exophytic nodule of the maxillary gingiva in a young male, and (B) a large irregular nodule of the mandibular gingiva reaching from the lingual to the labial side, with loss of the left lower canine in a pregnant female

(the latter two when occurring in the gingiva during pregnancy). This spectrum of synonyms creates confusion and for this reason, we will only be using the two terms best known: the PG and $\mathrm{LCH}$.

\section{CLINICAL FEATURES}

\section{PYOGENIC GRANULOMA}

PG is an exophytic, erythematous mass arising from the mucous membranes of the oral cavity (Figure 2). Its surface can be smooth or lobulated, and sometimes ulcerated $[2,5-8]$. The most frequent appearance of the lesion is a solitary painless lesion, which tends to bleed upon touching $[2,5,7]$. The lesion is fast growing and develops between 3 to 5 months [2,5-7]. Other symptoms reported are a burning sensation, halitosis, mobility of associated teeth, bone loss, lip biting, submandibular lymphadenopathy, and occasional pain [7]. The mass is more often pedunculated than sessile [2, 6-9]. The lesion is primarily located on the anterior maxillary gingiva. Other areas affected can be the other gingival sites, lips, tongue, buccal mucosa, and palate $[2,5-9]$. The size of the lesion varies between 0.3 to $6 \mathrm{~cm}$ in diameter, with a mean of 1.1 to $1.5 \mathrm{~cm}[2,6,9]$.

PG can occur at any age, with a peak incidence between 10 and 40 years. There is a clear gender predilection with a strong female predominance of 69 to $79 \%$ [2, 5-7, 9]. They account for 20 to $30 \%$ of all reactive hyperplastic lesions presenting in the oral cavity, 40 to $60 \%$ of all gingival lesions, and 3 to $7 \%$ of all lesions of the oral cavity $[10,11]$. The incidence rate calculated in one study was 3.2\% [2]. Caucasians are more frequently affected than Africans [2]. More than 4 out of 5 patients (85\%) report a known precipitating factor, allowing a distinction from the LCH [9].
Over $8 \%$ of all PGs are found in pregnant women $[7,12]$. The occurrence is related to pregnancy duration, with the most PGs' seen in the third trimester. Women under 25 years of age most commonly develop PG in the first trimester, whereas in an older age group, they develop mostly in the third trimester of pregnancy [6].

\section{LOBULAR CAPILLARY HEMANGIOMA}

Like the PG, the oral LCH is also a red to brown colored papule, lobulated or smooth surfaced. Ulceration occurs in more than $88 \%$ of cases [4]. It is also a fast-growing lesion, with a size ranging from 0.2 to $1.5 \mathrm{~cm}$ and a mean diameter of $0.8 \mathrm{~cm}$, making it slightly smaller than the PG $[4,9]$. In the oral cavity, it is located mostly on the maxillary gingiva, followed by the tongue, labial mucosa, buccal mucosa, mandibular gingiva, and palate $[4,9]$. As in PG, bleeding is a common clinical manifestation [4]. The lesion is more often sessile than pedunculated $[4,9]$.

The lesion can also occur at any age, but the peak incidence of the LCH is in the $6^{\text {th }}$ and $7^{\text {th }}$ decade of life, which makes this lesion more common in an elderly population than does the PG $[4,9]$. There is also a female predominance, but less marked than with the PG, with the percentage of female patients ranging from 57 to $65 \%$ of cases $[4,9]$. In only 20 to $29 \%$ of cases was a known precipitating factor present (e.g., overhanging edges of restauration or biting), in contrast to $85 \%$ of PG cases [9]. This contributes to the hypothesis that the pathogenetic mechanism of both lesions are different. An overview of the clinical differences between the PG and $\mathrm{LCH}$ is provided in Table 3.

While PG and pregnancy may be related, LCH may show some association with vascular malformations. Indeed, $16 \%$ of LCHs' are associated with arteriove- 
TABLE 3. Comparison of clinical features between pyogenic granuloma and lobular capillary hemangioma

\begin{tabular}{|c|c|c|}
\hline Parameter & Pyogenic granuloma & Lobular capillary hemangioma \\
\hline \multirow[t]{2}{*}{ Macroscopic appearance } & \multicolumn{2}{|c|}{$\begin{array}{l}\text { Exophytic red erythematous nodule arising from the oral mucosa, } \\
\text { with or without ulceration }\end{array}$} \\
\hline & Pedunculated $>$ sessile & Sessile $>$ pedunculated \\
\hline Symptoms & \multicolumn{2}{|c|}{$\begin{array}{l}\text { Symptomatic but painless lesion, } \\
\text { bleeding is common }\end{array}$} \\
\hline Location & \multicolumn{2}{|c|}{ Mostly on maxillary gingiva } \\
\hline Peak age incidence & $10-40 \mathrm{yr}$. & $50-70 \mathrm{yr}$. \\
\hline Size & $\begin{array}{c}0.3-6.0 \mathrm{~cm} \\
\text { Mean, } 1.1-1.5 \mathrm{~cm}\end{array}$ & $\begin{array}{l}0.2-1.5 \mathrm{~cm} \\
\text { Mean, } 0.8 \mathrm{~cm}\end{array}$ \\
\hline Gender predilection & Female +++ & Female + \\
\hline Precipitating factor present & $80 \%$ & $20-29 \%$ \\
\hline Association & Pregnancy & Vascular malformations \\
\hline
\end{tabular}

nous malformations [13]. Again, a distinct pathogenetic mechanism is hereby suggested.

\section{HISTOLOGY}

\section{PYOGENIC GRANULOMA}

The reactive PG can be divided into two distinct areas, such as an ulcerative area, sometimes with bacterial colonization [7], and a central area (Figure 3). The central area consists of small radially organized capillary proliferations resembling granulation tissue, although some authors also report a lobular arrangement $[2,8,14]$. It is comprised of both small vessels with tiny lumina and large thin walled vessels with larger lumina, lined by flat endothelium $[7,9,15]$. The stroma is edematous and richly permeated by a mixed chronic and acute inflammatory infiltrate $[2,3,7-9,16]$. Sometimes the stroma takes on a myxoid appearance $[3,7]$. In chronic lesions, foci of abundant collagen and fibrosis can be seen in the stroma, with reduced vascularity of the area $[2,3,7,9,15$, 16]. A great number of mast cells may be present in this fibrous region [17].

The ulcerative area of the PG consists of a superficial layer of fibrin with entrapped neutrophils. Below that, there are vessels of various sizes and various proportions of cells, composing the vascular walls. The stroma is edematous, with a mixed inflammatory infiltrate. Mean vessel diameter is around $19 \mu \mathrm{m}$, which is markedly larger than in the underlying area $[4,9]$.

\section{LOBULAR CAPILLARY HEMANGIOMA}

The central, or lobular, area of an LCH, consists of characteristic lobular proliferations of microvasculature forming tiny lumina $[4,9,18]$. The vessels are uniform in size and contain a similar proportion of cells forming the vascular wall, which is mostly composed of ovoid to spindle-shaped cells (Figure 3) [4, 12]. A variable number of mitotic figures can be found throughout this region [9]. The stroma is edematous, with occasionally a lymphocytic infiltrate in a superficial lobule [12]. Extensive necrosis can sometimes be seen throughout the lesion [19]. Mean vessel diameter of the lobular area is around 9 microns [4].

The ulcerative area of the $\mathrm{LCH}$ is identical to PG.

\section{PATHOGENESIS}

Because both lesions have distinct clinical and histological appearances, the pathogenic mechanisms are likely to be different. PGs' have an obvious predilection for (pregnant) women and have a marked inflammatory infiltrate, factors, which underlie its pathogenesis. In contrast, no inflammatory infiltrate or provoking factors are seen in LCH, suggesting that its development is rather spontaneous. However, the pathogenic mechanisms are likely to overlap between both lesions.

\section{PYOGENIC GRANULOMA}

In the PG, the major predisposing factors are female gender and pregnancy, and the primary precipitating factors are chronic low-grade irritation or minor injury. The high levels of estrogen and progesterone in (pregnant) women also contribute to the formation of the PG. An extensive review of pathogenic mechanisms can be found in a paper by Kamar et al. [20]. In conclusion, they all contribute to the characteristic histological appearance of this lesion: increased vascular permeability induced by estrogen leads to stromal edema, penetration of bacteria aids the formation of a mixed inflammatory infiltrate potentiated by estrogen, the effects of VEGF 

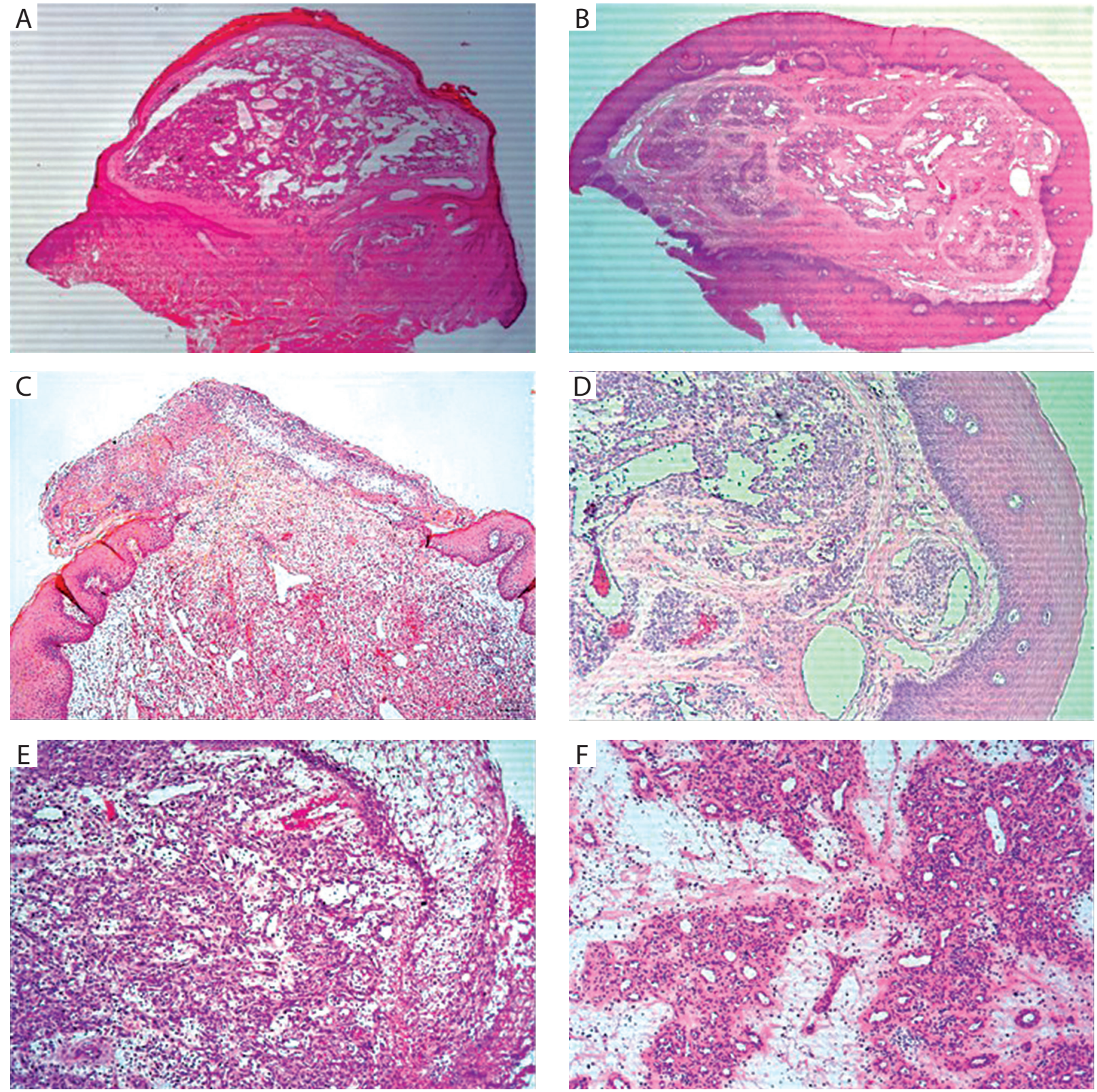

FIGURE 3. Comparison of the histological features of the pyogenic granuloma $(P G)(A, C, E)$ and the lobular capillary hemangioma (LCH) (B, D, F). A) PG at low magnification with chaotically organized capillaries. B) LCH at low magnification, with the clear lobular arrangement with fibrous septae. C) Ulcerated PG with a mixed inflammatory infiltrate and underlying radially organized capillaries. D) LCH with more uniform small capillaries and a lymphocytic inflammatory infiltrate. E) PG at high magnification with densely packed capillaries. F) LCH at high magnification, with organized lobules of uniform capillaries

(vascular endothelial growth factor) and HIF-1a (hypoxia-inducible factor-1 $\alpha$ ) are increased proliferation and migration of vascular endothelial cells, and pericytes and mast cells in the inflammatory infiltrate lead to fibrosis in chronic lesions. During this maturation, progressive fibrous replacement may lead to the development of a fibrous epulis or fibroepithelial polyp. The percentage of fibrous epulis (and fibroepithelial polyps) that develop from pre-existing PG is unknown.

Interestingly, 'estrogen-receptors' have not been demonstrated in PG (or LCH) [21, 22]. However, as de- scribed above, estrogen is likely to play a role in the pathogenesis. In the same study, 'progesterone-receptors' have been found in only $43 \%$ of the lesions.

\section{LOBULAR CAPILLARY HEMANGIOMA}

The pathogenesis of $\mathrm{LCH}$ and $\mathrm{PG}$ as separate lesions has not yet been presented in the literature. We propose that bone marrow stem cells, induced by an unknown stimulus, differentiate into endothelial cells, pericytes, and ovoid cells. The newly formed ovoid cells play a cen- 
tral role in the further development of the lesion. First, they interact with the pericytes to modulate neovascularization [23]. Secondly, the ovoid cells express the tie-2 receptor, a transmembrane tyrosine-kinase receptor for angiopoietine-1 (ang-1) and angiopoietine-2 (ang-2). In general, ang-1 (agonist) stabilizes the angiogenic process and ang-2 (antagonist), induces neovascularization or angiogenesis. Ang-2 induces overexpression of the wt-1 protein (Wilms' tumor-1 protein) in the cytoplasm of ovoid cell [24]. This causes proliferation of the vascular smooth muscle cells, endothelial cells, and ovoid cells giving rise to the lesion [25].

Finally, LCHs are known to arise from vascular malformations, such as port-wine stains or arteriovenous malformations, which suggests that these malformations provide a favorable environment for LCHs to develop in. The tissues in and around vascular malformations are thought to be in a constant hypoxic state, due to ineffective delivery of oxygen through the malformed vasculature. This hypoxia, like in the development of the PG, induces the angiogenic factors VEGF and HIF-1 $\alpha$, and thus neovascularization [26].

\section{DIFFERENTIAL DIAGNOSIS}

The differential diagnosis for PG and LCH is briefly discussed. The lesions mentioned may resemble PG and LCH microscopically, and therefore are to be considered during the establishment of a final diagnosis (Figure 4).

Two immunohistochemical stains are used to identify the nature of the lesion that is presented. D2-40 (podoplanin) differentiates between lesions from lymphatic origin (positive stain) and non-lymphatic origin (negative stain), and glut-1 (glucose transporter-1) discriminates between hemangiomas (positive stain), PG, and LCH (negative stain):

- Fibroma [7], described as a fibrous epulis if present on the gingiva, and a fibroepithelial polyp if located on the lining of the oral mucosa. Several most likely represent end stage of a PG, with fibrous replacement of the vascular core.

- Granulation tissue formation is common and a nonspecific reaction to the tissue damage [4].

- Peripheral ossifying fibromas are located only on the gingiva and resemble fibrous epulis clinically but are distinguished microscopically by the active deposition of bone in the core of the lesion. However, osteopontin is also expressed in some PG, so that its histopathology may mimic that of the peripheral ossifying fibroma [16].

- Peripheral giant cell granulomas [7] are located in the gingiva and represent the peripheral variant of the central type, which is situated in the jawbone.

- Kaposi Sarcoma, tumor stage $[15,27]$. Bundles of spindle cells and pseudo-vascular blood-filled spaces are seen. However, there is an invasive rather than a lobular growth pattern. The lesion stains positive for HHV-8 (human herpes virus-8) and D2-40, and negative for vWF (von Willebrand factor) and SMA (smooth muscle actin).

- Hemangiomas $[7,14]$ present as adult or juvenile types [4] and may be associated with inflammation, which complicates the differential diagnosis [28]. Capillary hemangiomas contain capillary-sized endothelial lined vessels and cavernous hemangiomas larger thin walled blood vessel spaces.

- Tufted angioma and Kaposiform hemangioendothelioma [29] are lesions on a spectrum from less aggressive (tufted angioma) to very aggressive (Kaposiform hemangioendothelioma). Lobules are D2-40 negative.

- Hemangiopericytoma (also known as a solitary fibrous tumor) consists of CD34 positive cells forming stag-horn shaped blood vessels in a typical reticulin fiber network.

- Angiofibroma is a rare nasopharyngeal tumor often associated with excessive nose bleeding.

- Angiosarcoma shows a high mitotic index and atypia, but an invasive growth pattern rather than an exophytic growth pattern, such as PG and LCH.

- Bacillary angiomatosis [30] is caused by an infection with Bartonella henselae, and is characterized by a lobular proliferation of endothelial cells resembling a PG with endothelial atypia, neutrophils with leukocytoclasis, and stainable bacteria.

\section{TREATMENT}

For PG, surgical excision (shaving excision and cautery) under local anesthesia, together with removal of local irritants is the standard therapy $[2,6,7]$. Excision down to the periosteum minimizes the risk of recurrence [2]. Recurrence rates vary from 8 to $15 \%$ and can occur up to 5 years after surgery, with maxillary lesions and lesions in female patients recurring more frequently $[2,7]$. Specific treatment options for LCHs have been less exploited, but as for PG, surgical excision is the most performed.

Many other treatment options have been explored, mainly for cutaneous or conjunctival PG. A brief overview is given below.

- Monoethanolamide oleate is known to induce damage to the vascular endothelium resulting in thrombus formation. In one study, this caused regression of all PG [31].

- Alitretinoin gel $0.1 \%$ is an FDA-approved treatment for localized Kaposi sarcoma, and in one study, it has been proven effective in the treatment of PG, possibly due to some histologic similarities [32].

- Low-dose plaque brachytherapy has been reported effective in the treatment of a chronically recurring conjunctival PG [33].

- Imiquimod $5 \%$ cream provides a 75 to $99 \%$ size reduction of PG, but with possible systemic side-effects 

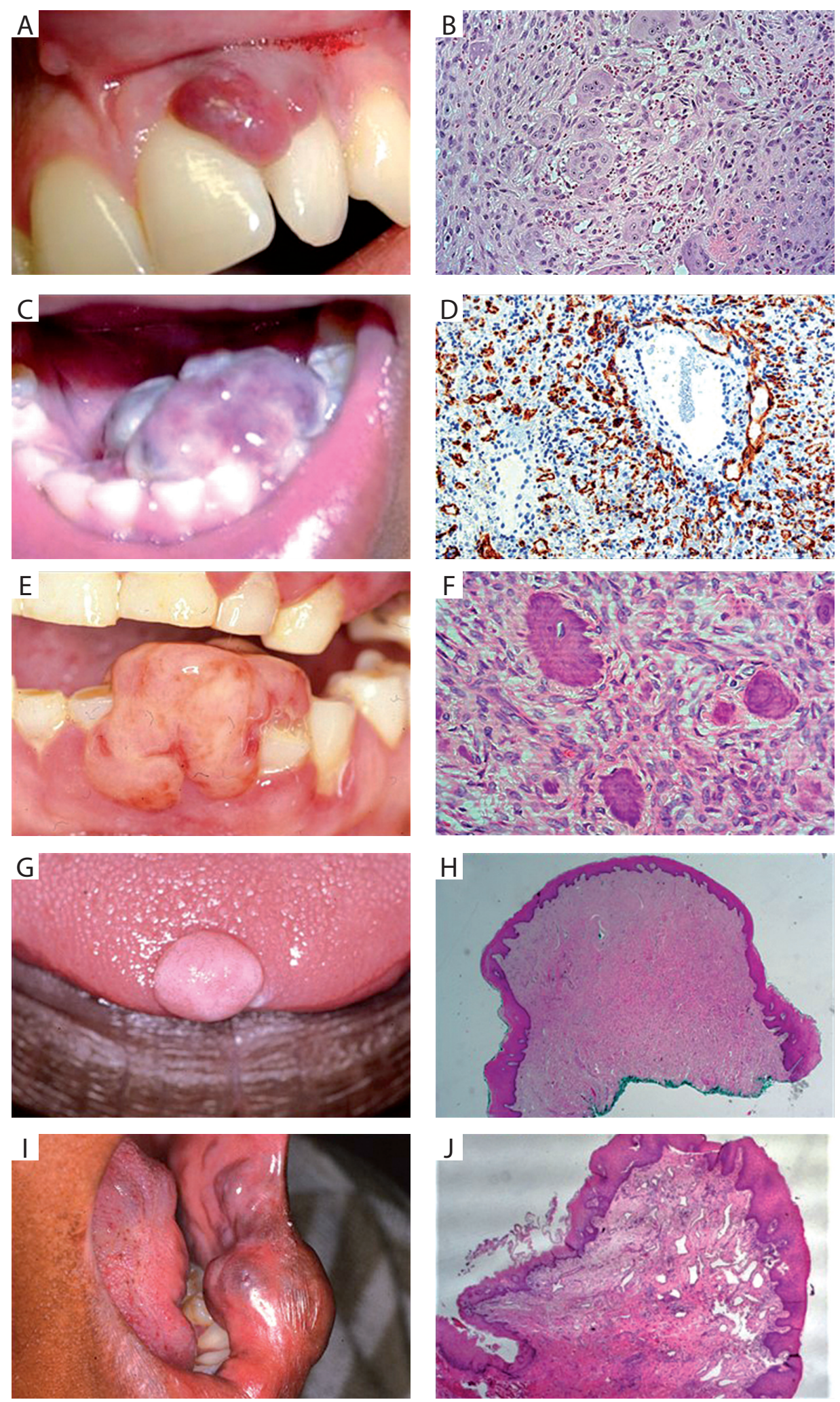

FIGURE 4. Paired clinical and histological images of differential diagnoses to consider for a pyogenic granuloma or lobular capillary hemangioma. A, B) Peripheral giant cell granuloma: note the distinctive blue color of the erosion. Multinuclear giant cells are a typical feature of this lesion. C, D) Kaposi sarcoma, with a nodular surface indicating tumor stage. On histological examination there are D2-40-positive vascular spaces. E, F) Peripheral ossifying fibroma, clinically resembling a fibrous epulis. Foci of ossification are seen on histological examination. G, H) Fibroepithelial polyp on the tip of the tongue. Note the broad-based polypoid appearance and light pink color resembling that of normal masticatory mucosa. Microscopically, the characteristic fibrous connective tissue core is demonstrated. I, J) Hemangioma of the lower lip with the typical blue color showing capillary-sized endothelial lined vessels on histological examination 
(fever, nausea, diarrhea). This regression results from the anti-angiogenic and apoptotic effects of imiquimod [34].

- FSH (follicle stimulating hormone) antagonists are hypothesized to prevent recurrence after primary treatment [35].

- Timolol and other beta-adrenergic blockers have been used to treat infantile hemangiomas as well as some PG (only about $50 \%$ of PGs express $\beta$-adrenergic receptors). Initial effects are due to vasoconstriction, but significant regression takes more than 6 weeks. $\beta$-blockage is believed to inhibit angiogenic factors, and some patients show continuation of regression even after they stop the therapy. This suggests that even a short regimen of beta-blocking agents can induce apoptosis and regression of the lesion [36].

- Injection of absolute $99.5 \%$ ethanol resulted in regression of all PG without scarring. Ethanol dehydrates the lesion and causes cell necrosis, resulting in thrombosis and ischemia of the lesion [37].

\section{CONCLUSIONS}

Based on a scoping review of the available literature, we found several clinical and histological differences between the PG and the LCH. Compared to the $\mathrm{LCH}$, the PG occurs more in the middle-aged range, has a clearer predilection for (pregnant) females, and has more frequently a known triggering factor in the history. Histologically, PGs appear mostly as radially organized capillaries (while LCHs have a lobular pattern), have a mixed inflammatory infiltrate (compared to the occasional lymphocytic infiltrate in LCHs), and can undergo fibrosing transformation when chronic, which is not seen in LCHs.

When first described, the PG was considered a form of exuberant granulation tissue. Later, some lesions were found to show a lobular pattern of capillary proliferation, giving rise to the term "lobular capillary hemangioma" as the underlying histological presence of the lesion. After that, there was mixed use of the names for both the granulation tissue-like PG and the vascular neoplasm-like LCH. The latest WHO classification of head and neck tumors (2017) states that both lesions reside under term "pyogenic granuloma", with LCH used as a synonym, and both being considered hemangiomas. In contrast, the International Society for the Study of Vascular Anomalies (ISSVA) in their latest classifications (2018) states that both lesions are synonyms and are considered benign vascular tumors. However, given the clinical behavior of the PG (reactive lesion to microtrauma, stops growing at certain size, thus not being a tumor strictu sensu), classifying this under the term "tumors" may lead to even more confusion. We propose that both lesions again to be considered as distinct for two reasons. First, there are the proposed differences in clinical features, histology, and etiopathogenesis. Sec- ond, disconnecting the two lesions makes interpretation of the existing literature much easier.

The limitation of this study is that it is a review of articles, which not all make a clear distinction between the PG and the LCH. Also, histological descriptions were often compact, so misclassification of the lesions cannot be ruled out. Because of this, statistical analysis of the data was impossible, nor would it have been conclusive. However, because of the large number of patients in the included articles, some broad perspectives have become clear, which can and need to be addressed in future research.

\section{CONFLICT OF INTEREST}

The authors declare no potential conflicts of interest with respect to the research, authorship, and/or publication of this article.

\section{References}

1. Airey FS. Pyogenic granuloma of the chin, associated with and dependent upon a dental alveolar abscess. Proc R Soc Med 1943; 36: 293-294.

2. Gordon-Nunez MA, de Vasconcelos Carvalho M, Benevenuto TG, Lopes MFF, Silva LMM, Galvao HC. Oral pyogenic granuloma: a retrospective analysis of 293 cases in a Brazilian population. J Oral Maxillofac Surg 2010; 68: 2185-2188.

3. Mills SE, Cooper PH, Fechner RE. Lobular capillary hemangioma: the underlying lesion of pyogenic granuloma. A study of 73 cases from the oral and nasal mucous membranes. Am J Surg Pathol 1980; 4: 470-479.

4. Toida M, Hasegawa T, Watanabe F, et al. Lobular capillary hemangioma of the oral mucosa: clinicopathological study of 43 cases with a special reference to immunohistochemical characterization of the vascular elements. Pathol Int 2003; 53: 1-7.

5. Maturana-Ramirez A, Adorno-Farias D, Reyes-Rojas M, FariasVergara M, Aitken-Saavedra J. A retrospective analysis of reactive hyperplastic lesions of the oral cavity: study of 1149 cases diagnosed between 2000 and 2011, Chile. Acta Odontol Latinoam 2015; 28: 103-107.

6. Cardoso JA, Spanemberg JC, Cherubini K, Figueiredo MAZ de, Salum FG. Oral granuloma gravidarum: a retrospective study of 41 cases in Southern Brazil. J Appl Oral Sci 2013; 21: 215-218.

7. Krishnapillai R, Punnoose K, Angadi PV, Koneru A. Oral pyogenic granuloma - a review of 215 cases in a South Indian Teaching Hospital, Karnataka, over a period of 20 years. Oral Maxillofac Surg 2012; 16: 305-309.

8. Diaz-Guzman LM, Castellanos-Suarez JL. Lesions of the oral mucosa and periodontal disease behavior in pregnant patients. Med Oral Patol Oral Cir Bucal 2004; 9: 433-434.

9. Epivatianos A, Antoniades D, Zaraboukas T, et al. Pyogenic granuloma of the oral cavity: comparative study of its clinicopathological and immunohistochemical features. Pathol Int 2005; 55: 391-397.

10. Buchner A, Shnaiderman-Shapiro A, Vered M. Relative frequency of localized reactive hyperplastic lesions of the gingiva: a retrospective study of 1675 cases from Israel. J Oral Pathol Med 2010; 39: 631-638.

11. Al-Khateeb TH. Benign oral masses in a Northern Jordanian population - a retrospective study. Open Dent J 2009; 3: 147-153.

12. Saravana GHL. Oral pyogenic granuloma: a review of 137 cases. Br J Oral Maxillofac Surg 2009; 47: 318-319.

13. Chen $\mathrm{D}, \mathrm{Hu} \mathrm{X}$, Lin $\mathrm{X}$, et al. Nodules arising within port-wine stains: a clinicopathologic study of 31 cases. Am J Dermatopathol 2011; 33: 144-151 
14. Freitas TMC, Miguel MCC, Silveira EJD, Freitas RA, Galvao HC. Assessment of angiogenic markers in oral hemangiomas and pyogenic granulomas. Exp Mol Pathol 2005; 79: 79-85.

15. Ramirez-Amador V, Martinez-Mata G, Gonzalez-Ramirez I, AnayaSaavedra G, de Almeida OP. Clinical, histological and immunohistochemical findings in oral Kaposi's sarcoma in a series of Mexican AIDS patients. Comparative study. J Oral Pathol Med 2009; 38: 328-233.

16. Elanagai R, Veeravarmal V, Nirmal RM. Osteopontin expression in reactive lesions of gingiva. J Appl Oral Sci 2015; 23: 26-32.

17. Ferreira SV, Xavier FCA, Freitas M da CA de, et al. Involvement of mast cells and microvessels density in reactive lesions of oral cavity: a comparative immunohistochemical study. Pathol Res Pract 2016; 212: 761-766

18. Meijer-Jorna LB, van der Loos CM, Teeling P, et al. Proliferation and maturation of microvessels in arteriovenous malformations - expression patterns of angiogenic and cell cycle-dependent factors. J Cutan Pathol 2012; 39: 610-620.

19. Lim YH, Douglas SR, Ko CJ, et al. Somatic activating RAS mutations cause vascular tumors including pyogenic granuloma. J Invest Dermatol 2015; 135: 1698-1700.

20. Kamal R, Dahiya P, Puri A. Oral pyogenic granuloma: various concepts of etiopathogenesis. J Oral Maxillofac Pathol 2012; 16: 79-82.

21. Park SH, Lee JH, Tak MS, Lee HJ, Choi HJ. A research of pyogenic granuloma genesis factor with immunohistochemical analysis. J Craniofac Surg 2017; 28: 2068-2072.

22. Kamiyama K, Kinjo T, Chinen $\mathrm{K}$, et al. Human herpesvirus 8 (HHV8) sequence variations in HHV8 related tumours in Okinawa, a subtropical island in southern Japan. J Clin Pathol 2004; 57 : 529-535.

23. Sato H, Takeda Y, Satoh M. Expression of the endothelial receptor tyrosine kinase Tie2 in lobular capillary hemangioma of the oral mucosa: an immunohistochemical study. J oral Pathol Med 2002; 31: 432-438.

24. Lawley LP, Cerimele F, Weiss SW, et al. Expression of Wilms tumor 1 gene distinguishes vascular malformations from proliferative endothelial lesions. Arch Dermatol 2005; 141: 1297-1300.

25. Small TW, Bolender Z, Bueno C, et al. Wilms' tumor 1-associating protein regulates the proliferation of vascular smooth muscle cells. Circ Res 2006; 99: 1338-1346.

26. Forsythe JA, Jiang BH, Iyer NV, et al. Activation of vascular endothelial growth factor gene transcription by hypoxia-inducible factor 1. Mol Cell Biol 1996; 16: 4604-4613.

27. Patel RM, Goldblum JR, Hsi ED. Immunohistochemical detection of human herpes virus- 8 latent nuclear antigen- 1 is useful in the diagnosis of Kaposi sarcoma. Mod Pathol 2004; 17: 456-460.

28. Leon-Villapalos J, Wolfe K, Kangesu L. GLUT-1: an extra diagnostic tool to differentiate between haemangiomas and vascular malformations. Br J Plast Surg 2005; 58: 348-352.

29. Debelenko LV, Perez-Atayde AR, Mulliken JB, Liang MG, Archibald TH, Kozakewich HPW. D2-40 immunohistochemical analysis of pediatric vascular tumors reveals positivity in kaposiform hemangioendothelioma. Mod Pathol 2005; 18: 1454-1460.

30. Tucci E, Della Rocca C, Santilli F. Localized bacillary angiomatosis in the oral cavity: observations about a neoplasm with atypical behavior. Description of a case and review of the literature. Minerva Stomatol 2006; 55: 67-75.

31. Matsumoto K, Nakanishi H, Seike T, Koizumi Y, Mihara K, Kubo Y. Treatment of pyogenic granuloma with a sclerosing agent. Dermatol Surg 2001; 27: 521-523.

32. Maloney DM, Schmidt JD, Duvic M. Alitretinoin gel to treat pyogenic granuloma. J Am Acad Dermatol 2002; 47: 969-970.

33. Gunduz K, Shields CL, Shields JA, Zhao DY. Plaque radiation therapy for recurrent conjunctival pyogenic granuloma. Arch Ophthalmol 1998; 116: 538-539.

34. Mao X, Wang J, Yan J. Topical imiquimod treatment of cutaneous vascular disorders in pediatric patients: clinical evaluation on the efficacy and safety. J Zhejiang Univ Sci B 2012; 13: 745-750.
35. Maclellan RA, Vivero MP, Purcell P, et al. Expression of folliclestimulating hormone receptor in vascular anomalies. Plast Reconstr Surg 2014; 133: 344e-351e.

36. Wine Lee L, Goff KL, Lam JM, Low DW, Yan AC, Castelo-Soccio L. Treatment of pediatric pyogenic granulomas using beta-adrenergic receptor antagonists. Pediatr Dermatol 2014; 31: 203-207.

37. Ichimiya M, Yoshikawa Y, Hamamoto Y, Muto M. Successful treatment of pyogenic granuloma with injection of absolute ethanol. J Dermatol 2004; 31: 342-344. 